PROCEEDINGS OF THE

AMERICAN MATHEMATICAL SOCIETY

Volume 139, Number 6, June 2011, Pages 2271-2279

S 0002-9939(2010)10640-8

Article electronically published on November 29, 2010

\title{
KLYACHKO MODELS OF $p$-ADIC SPECIAL LINEAR GROUPS
}

\author{
JOSHUA M. LANSKY AND C. RYAN VINROOT
}

(Communicated by Wen-Ching Winnie Li)

\begin{abstract}
We study Klyachko models of $\operatorname{SL}(n, F)$, where $F$ is a nonArchimedean local field. In particular, using results of Klyachko models for GL $(n, F)$ due to Heumos, Rallis, Offen and Sayag, we give statements of existence, uniqueness, and disjointness of Klyachko models for admissible representations of $\mathrm{SL}(n, F)$, where the uniqueness and disjointness are up to specified conjugacy of the inducing character, and the existence is for unitarizable representations in the case $F$ has characteristic 0 . We apply these results to relate the size of an $L$-packet containing a given representation of $\operatorname{SL}(n, F)$ to the type of its Klyachko model, and we describe when a self-dual unitarizable representation of $\mathrm{SL}(n, F)$ is orthogonal and when it is symplectic.
\end{abstract}

\section{INTRODUCTION}

Let $F$ be a field, let $U_{m}(F)$ denote the group of $m$-by- $m$ unipotent upper triangular matrices over $F$, and let $M_{m, l}(F)$ be the set of $m$-by-l matrices over $F$ (not necessarily invertible). For each integer $k$ satisfying $0 \leq 2 k \leq n$, define the subgroup $G_{k}$ of $\mathrm{GL}(n, F)$ by:

$$
G_{k}=\left\{\left(\begin{array}{cc}
N & X \\
& S
\end{array}\right) \mid N \in U_{n-2 k}, S \in \operatorname{Sp}(2 k, F), X \in M_{n-2 k, 2 k}(F)\right\} .
$$

Here we define, for $k>0$, the symplectic group $\operatorname{Sp}(2 k, F)$ to be the stabilizer of the form corresponding to the skew-symmetric matrix $J_{k}=\left({ }_{1_{k}}-1_{k}\right)$. Fix a nontrivial additive character $\theta: F^{+} \rightarrow \mathbb{C}$, and for each $k$, define a character $\psi_{k}$ on $G_{k}$ as follows:

If $g \in G_{k}, g=\left(\begin{array}{cc}N & X \\ & S\end{array}\right)$, and $N=\left(a_{i j}\right)$, then define $\psi_{k}(g)=\theta\left(\sum_{i=1}^{n-2 k-1} a_{i, i+1}\right)$.

In other words, $\psi_{k}$ is only nontrivial on the unipotent factor of $G_{k}$. When $n=$ $2 m$, then $\psi_{m}$ is just the trivial character on the subgroup $G_{m}=\operatorname{Sp}(2 m, F)$, and when $k=0, \psi_{k}$ is a nondegenerate character of the unipotent subgroup $U_{n}(F)$ of $\mathrm{GL}(n, F)$.

Suppose that $F=\mathbb{F}_{q}$ is a finite field, let $G=\operatorname{GL}\left(n, \mathbb{F}_{q}\right)$, and for each $k, 0 \leq 2 k \leq$ $n$, define the induced representation $T_{k}=\operatorname{Ind}_{G_{k}}^{G}\left(\psi_{k}\right)$. Klyachko [8] claimed that

Received by the editors September 3, 2009 and, in revised form, June 10, 2010.

2000 Mathematics Subject Classification. Primary 22E50.

Key words and phrases. Klyachko model, p-adic special linear group, multiplicity one, $L$ packets, self-dual representations. 
for any complex irreducible representation $(\pi, V)$ of $G, \operatorname{dim}_{\mathbb{C}} \operatorname{Hom}_{G}\left(\pi, T_{k}\right) \leq 1$ for every $k$, and there exists a unique $k, 0 \leq 2 k \leq n$, such that $\operatorname{dim}_{\mathbb{C}} \operatorname{Hom}_{G}\left(\pi, T_{k}\right)=1$. After Klyachko's original paper, Inglis and Saxl [7] gave the first complete proof to Klyachko's claim.

We call an embedding of the representation $(\pi, V)$ in the induced representation $T_{k}$ a Klyachko model of the representation $\pi$. Klyachko's original result states that every irreducible representation of $\operatorname{GL}\left(n, \mathbb{F}_{q}\right)$ has a unique Klyachko model, and in particular, all of the induced representations $T_{k}$ are multiplicity-free, and $T_{k}$ and $T_{l}$ have no isomorphic subrepresentations when $k \neq l$.

Now consider the case that $F$ is a non-Archimedean local field, with $G=$ $\mathrm{GL}(n, F)$. For each $k, 0 \leq 2 k \leq n$, define the representation $T_{k}$ by

$$
T_{k}=\operatorname{Ind}_{G_{k}}^{G}\left(\psi_{k}\right),
$$

where Ind denotes the ordinary (nonnormalized) induced representation for a locally compact totally disconnected group. In this case, we have the following result on Klyachko models of representations of $\mathrm{GL}(n, F)$.

Theorem 1.1 (Heumos and Rallis, Offen and Sayag). Let $G=\mathrm{GL}(n, F)$, where $F$ is a non-Archimedean local field. Let $(\pi, V)$ be any irreducible admissible representation of $G$. We have the following:

(1) $\sum_{k=0}^{\lfloor n / 2\rfloor} \operatorname{dim}_{\mathbb{C}} \operatorname{Hom}_{H}\left(\pi, T_{k}\right) \leq 1$.

(2) If $F$ has characteristic 0 and $(\pi, V)$ is unitarizable, then there exists a unique $k$ such that $\operatorname{dim}_{\mathbb{C}} \operatorname{Hom}_{G}\left(\pi, T_{k}\right)=1$.

Heumos and Rallis [6] proved that, if $n=2 m$, then for any $\pi, \operatorname{dim}_{\mathbb{C}} \operatorname{Hom}_{H}\left(\pi, T_{m}\right)$ $\leq 1$; that is, any irreducible admissible representation has a unique symplectic model if one exists. They also proved that, in this case, the set of admissible representations of $\operatorname{GL}(n, F)$ which have symplectic models is disjoint with the set of representations which have Whittaker models. Finally, Heumos and Rallis proved statements (1) and (2) of Theorem 1.1 for $n \leq 4$ and conjectured that these statements hold for all $n$. Theorem 1.1 was proved completely by Offen and Sayag in a series of papers [9, 10, 11].

Now notice that the groups $G_{k}$ are also subgroups of the special linear group $\mathrm{SL}(n, F)$. In this paper, we study Klyachko models of the group $\operatorname{SL}(n, F)$ when $F$ is a non-Archimedean local field. Since there is more than one orbit of nondegenerate characters of the unipotent subgroup of $\operatorname{SL}(n, F)$, we must consider conjugates of the characters $\psi_{k}$ in (1.2) in these models. Our main result, Theorem 2.1, is the analogue of Theorem 1.1 for the special linear group. The main difference in the result is in the statement of uniqueness and disjointness of Theorem 2.1. where we can only obtain uniqueness and disjointness of Klyachko models up to conjugation of the character $\psi_{k}$ by an element of a certain group.

We give two applications of Theorem 2.1. In the first, Corollary 2.1, we relate the type of the Klyachko model of a representation of $\mathrm{SL}(n, F)$ to the size of the $L$-packet containing that representation. In the second, Corollary 3.1, we describe when a self-dual unitarizable representation of $\operatorname{SL}(n, F)$ is orthogonal and when it is symplectic. 


\section{KLYACHKO MODELS OF SPECIAL LINEAR GROUPS}

From now on, we let $F$ be a non-Archimedean local field, let $G=\mathrm{GL}(n, F)$, let $H=\mathrm{SL}(n, F)$, and let $G_{k}$ be as in (1.1) for each $k$ such that $0 \leq 2 k \leq n$. Note that $G \cong H \ltimes D$, where $D \cong F^{\times}$is the group of matrices of the form $\operatorname{diag}(x, 1, \ldots, 1)$ for $x \in F^{\times}$. We will often identify $G / H$ with $D$ and hence with $F^{\times}$. Note that $H$ contains each $G_{k}$, and $D$ normalizes $G_{k}$ whenever $0 \leq 2 k<n$.

Let $(\rho, W)$ be an irreducible admissible representation of $H$. By [14, Prop. 2.2], there is an $H$-embedding of $(\rho, W)$ as a direct summand of some irreducible admissible representation $(\pi, V)$ of $G$. From results in [2, 14, we know that if $\rho$ is unitarizable, then $(\pi, V)$ can also be taken to be unitarizable.

Given any $g \in G$, define ${ }^{g} \rho$ to be the representation of $H$ on $W$ given by ${ }^{g} \rho(h)=$ $\rho\left(g^{-1} h g\right)$. Denote by $G(\rho)$ the subgroup $\left\{\left.g \in G\right|^{g} \rho \cong \rho\right\}$ of $G$. By [14, Cor. 2.3], $G(\rho)$ is an open normal subgroup of finite index in $G$. Since $\rho$ is stable under conjugation by $G(\rho)$, we may extend $\rho$ to a representation of the group $G(\rho)$. We let $D(\rho)=D \cap G(\rho)$.

If $g \in G$ normalizes $G_{k}$, and $\psi$ is a character of $G_{k}$, denote by ${ }^{g} \psi$ the character $\alpha \mapsto \psi\left(g^{-1} \alpha g\right)$. In this case, if $g$ has image $x \in F^{\times}$under the map $G \mapsto G / H \cong$ $D \cong F^{\times}$, we have (in the notation of (1.2) $)$

$$
{ }^{g} \psi_{k}(g)=\theta\left(x^{-1} a_{1,2}+\sum_{i=2}^{n-2 k-1} a_{i, i+1}\right) \quad \text { when } 0 \leq 2 k<n-1 .
$$

If $n$ is odd and $2 k=n-1$, then $\psi_{k}$ is trivial, hence unaffected by conjugation. If $n$ is even and $2 k=n$, then $\psi_{k}$ is again trivial, although $G_{k}=\operatorname{Sp}(2 k, F)$ is no longer normalized by nontrivial elements of $D$. In this case, given $\gamma \in D, \gamma{ }^{\gamma}{ }_{k}=\gamma G_{k} \gamma^{-1}$ is the symplectic group defined by the skew-symmetric matrix $\gamma J_{k} \gamma$. So, in general, ${ }^{\gamma} \psi_{k}$ is a character of ${ }^{\gamma} G_{k}$, and $G_{k}={ }^{\gamma} G_{k}$ in all cases except when $2 k=n$. We first prove a result which relates representations of $H, G(\rho)$, and $G$.

Lemma 2.1. Let $(\rho, W)$ be an irreducible admissible representation of $H$ and let $(\pi, V)$ be an irreducible admissible representation of $G$ that contains $(\rho, W)$ upon restriction. Consider $(\rho, W)$ as an irreducible representation of $G(\rho)$. Then $\operatorname{Ind}_{G(\rho)}^{G}(\rho) \cong \pi$.

Proof. Since the restriction of $(\pi, V)$ to $G(\rho)$ contains $(\rho, W)$, it follows that $\operatorname{Ind}_{G(\rho)}^{G}(\rho)$ is contained in

$$
\operatorname{Ind}_{G(\rho)}^{G}(\pi)=\operatorname{Ind}_{G(\rho)}^{G}(\pi \otimes \mathbf{1})=\pi \otimes \operatorname{Ind}_{G(\rho)}^{G}(\mathbf{1}),
$$

which is completely reducible. Therefore, $\operatorname{Ind}_{G(\rho)}^{G}(\rho)$ is completely reducible, and the number of irreducible components therein is given by

$$
\operatorname{dim}_{\mathbb{C}}\left(\operatorname{End}_{G}\left(\operatorname{Ind}_{G(\rho)}^{G}(\rho)\right)\right) .
$$

An application of Mackey's theorem [1, Exer. 4.5.5] shows that this dimension is 1.

The following lemma relates models for representations of $G$ with those of $H$.

Lemma 2.2. Let $(\rho, W)$ be an irreducible admissible representation of $H$ and let $(\pi, V)$ be an irreducible admissible representation of $G$ that contains $(\rho, W)$ upon restriction. Suppose that for some $\gamma \in D$ and some $k$ with $0 \leq 2 k \leq n,(\rho, W)$ embeds in $\operatorname{Ind}_{\gamma}^{H} G_{k}\left({ }^{\gamma} \psi_{k}\right)$. Then $(\pi, V)$ embeds in $T_{k}=\operatorname{Ind}_{\gamma}^{G} G_{k}\left({ }^{\gamma} \psi_{k}\right)=\operatorname{Ind}_{G_{k}}^{G}\left(\psi_{k}\right)$. 
Proof. Since $\rho$ embeds in $\operatorname{Ind}_{\gamma_{G_{k}}}^{H}\left({ }^{\gamma} \psi_{k}\right)$, we have by Frobenius reciprocity,

$$
\operatorname{Hom}_{G(\rho)}\left(\rho, \operatorname{Ind}_{\gamma G_{k}}^{G(\rho)}\left({ }^{\gamma} \psi_{k}\right)\right) \neq(0),
$$

where we view $\rho$ as a representation of $G(\rho)$ and have applied transitivity of induction in that $\operatorname{Ind}_{\gamma G_{k}}^{G(\rho)}\left({ }^{\gamma} \psi_{k}\right)=\operatorname{Ind}_{H}^{G(\rho)}\left(\operatorname{Ind}_{\gamma_{G_{k}}}^{H}\left({ }^{\gamma} \psi_{k}\right)\right)$. Note that if $\sigma_{1}$ and $\sigma_{2}$ are any two representations of $G(\rho)$ with $\operatorname{Hom}_{G(\rho)}\left(\sigma_{1}, \sigma_{2}\right)$ nonzero, an application of Mackey's theorem shows that $\operatorname{Hom}_{G}\left(\operatorname{Ind}_{G(\rho)}^{G}\left(\sigma_{1}\right), \operatorname{Ind}_{G(\rho)}^{G}\left(\sigma_{2}\right)\right)$ is also nonzero. Thus (2.1) implies that

$$
\operatorname{Hom}_{G(\rho)}\left(\operatorname{Ind}_{G(\rho)}^{G}(\rho), \operatorname{Ind}_{G(\rho)}^{G}\left(\operatorname{Ind}_{\gamma G_{k}}^{G(\rho)}\left({ }^{\gamma} \psi_{k}\right)\right)\right) \neq(0) .
$$

Using Lemma 2.1 and the transitivity of induction, this becomes

$$
\operatorname{Hom}_{G}\left(\pi, \operatorname{Ind}_{\gamma G_{k}}^{G}\left({ }^{\gamma} \psi_{k}\right)\right) \neq(0)
$$

as desired.

We now prove our main result.

Theorem 2.1. Let $H=\mathrm{SL}(n, F)$, where $F$ is a non-Archimedean local field. Let $(\rho, W)$ be an irreducible admissible representation of $H$. We have the following:

(1) For any collection $D_{\rho}$ of representatives of the cosets $D / D(\rho)$,

$$
\sum_{k=0}^{\lfloor n / 2\rfloor} \sum_{\gamma \in D_{\rho}} \operatorname{dim}_{\mathbb{C}} \operatorname{Hom}_{H}\left(\rho, \operatorname{Ind}_{\gamma G_{k}}^{H}\left({ }^{\gamma} \psi_{k}\right)\right) \leq 1 .
$$

(2) If $F$ has characteristic 0 and $\rho$ is unitarizable, then there exists a unique integer $k$ and a unique element $\gamma \in D_{\rho}$ such that $\operatorname{dim}_{\mathbb{C}} \operatorname{Hom}_{H}\left(\rho, \operatorname{Ind}_{\gamma_{G_{k}}}^{H}\left({ }^{\gamma} \psi_{k}\right)\right)$ $=1$.

Proof. Let $(\rho, W)$ be an irreducible admissible representation of $H$ and let $\gamma \in D$. Let $(\pi, V)$ be an irreducible admissible representation of $G$ in which $(\rho, W)$ embeds as a direct summand. If $\pi$ has no Klyachko model, then $\operatorname{Hom}_{H}\left(\rho, \operatorname{Ind}_{\gamma}^{H} G_{k}\left({ }^{\gamma} \psi_{k}\right)\right)$ must be trivial for all integers $k$ by Lemma 2.2. so (2.2) holds. Hence suppose from now on that $\pi$ embeds in $T_{k}$ for some integer $k$ with $0 \leq 2 k \leq n$.

Viewing $\rho$ as a representation of $G(\rho)$, Mackey's theorem implies that we have an isomorphism

$$
\operatorname{Hom}_{G}\left(\operatorname{Ind}_{G(\rho)}^{G}(\rho), T_{k}\right) \cong \bigoplus_{\delta \in G / G(\rho)} \operatorname{Hom}_{G_{k}}\left({ }^{\delta} \rho, \psi_{k}\right) .
$$

By Lemma 2.1 and Theorem 1.1, the dimension of the space on the left-hand side of $(2.3)$ is 1 .

Now consider the right-hand side of (2.3). The preceding paragraph implies that

$$
\sum_{\delta \in G / G(\rho)} \operatorname{dim}_{\mathbb{C}}\left(\operatorname{Hom}_{G_{k}}\left(\delta \rho, \psi_{k}\right)\right)=1 .
$$

Note that we may assume that our representatives for the cosets in $G / G(\rho)$ lie in $D$. Then we have, for any $\delta \in D$ in our set of coset representatives,

$$
\operatorname{Hom}_{G_{k}}\left({ }^{\delta} \rho, \psi_{k}\right)=\operatorname{Hom}_{\delta^{-1} G_{k}}\left(\rho,{ }^{\delta^{-1}} \psi_{k}\right)=\operatorname{Hom}_{H}\left(\rho, \operatorname{Ind}_{\delta^{-1} G_{k}}^{H}\left({ }^{\delta^{-1}} \psi_{k}\right)\right),
$$


where we have applied Frobenius reciprocity in the last equality. Letting $D_{\rho}$ be our set of coset representatives for $D / D(\rho)$, we can now rewrite (2.4) to obtain

$$
\sum_{\gamma \in D_{\rho}} \operatorname{dim}_{\mathbb{C}}\left(\operatorname{Hom}_{H}\left(\rho, \operatorname{Ind}_{\gamma_{G_{k}}}^{H}\left({ }^{\gamma} \psi_{k}\right)\right)\right)=1 .
$$

This forces $\operatorname{Hom}_{H}\left(\rho, \operatorname{Ind}_{\gamma_{G_{k}}}^{H}\left({ }^{\gamma} \psi_{k}\right)\right)$ to be one-dimensional for a unique choice of $\gamma \in D_{\rho}$.

Now suppose $\rho$ also embeds in $\operatorname{Ind}_{\delta_{G_{k}}}^{H}\left({ }^{\delta} \psi_{l}\right)$ for some integer $l$ and $\delta \in D$. Then Lemma 2.2 implies that $\pi$ also embeds in $T_{l}$, which forces $l=k$ by the uniqueness of the Klyachko model of $\pi$. This concludes the proof of (1) in the case that $\pi$ has a Klyachko model, and shows that in this case (2.2) is an equality.

Now suppose that $\rho$ is unitarizable. Note that statement (2) now follows from (1) as soon as it is shown that the representation $\pi$ has a Klyachko model. But by [14, Prop. 2.2, 2.7], we may assume that $\pi$ is itself unitarizable. Hence by Theorem 1.1. $\pi$ has a Klyachko model.

We will say that the representation $\rho$ of $H$ possesses a Klyachko model if $\operatorname{Hom}_{H}\left(\rho, \operatorname{Ind}_{\gamma_{G_{k}}}^{H}\left({ }^{\gamma} \psi_{k}\right)\right)$ is nontrivial for some integer $k$ and some $\gamma \in D$. Note that Theorem 2.1 can be adjusted to be a statement for Klyachko models for the finite group $\operatorname{SL}\left(n, \mathbb{F}_{q}\right)$, which sharpens the results in [15, Prop. 1].

We will need the following for an application of Theorem 2.1.

Lemma 2.3. Let $k$ be an integer, $0<2 k<n$, and let $d=(2 k, n)$. Suppose $\psi$ is a character of $G_{k}$ that is trivial on

$$
\left\{\left(\begin{array}{cc}
1_{n-2 k} & X \\
& 1_{2 k}
\end{array}\right) \mid X \in M_{n-2 k, 2 k}(F)\right\} .
$$

Then the equivalence class of $\operatorname{Ind}_{G_{k}}^{H}(\psi)$ is stable under conjugation by $D^{d}$.

Proof. Suppose $\delta \in D^{n}$, say $\delta=\operatorname{diag}\left(x^{n}, 1, \ldots, 1\right)$. Then

$$
\delta=\operatorname{diag}\left(x^{n-1}, x^{-1}, \ldots, x^{-1}\right) \operatorname{diag}(x, x, \ldots, x) \in H Z,
$$

where $Z$ is the center of $G$. Thus

$$
\delta\left(\operatorname{Ind}_{G_{k}}^{H}(\psi)\right) \cong \operatorname{Ind}_{G_{k}}^{H}(\psi) .
$$

Thus $D^{n}$ stabilizes the equivalence class of $\operatorname{Ind}_{G_{k}}^{H}(\psi)$.

Now suppose $\delta \in D^{n-2 k}$ so that $\delta=\operatorname{diag}\left(a^{n-2 k}, 1, \ldots, 1\right)$ for some $a \in F^{\times}$. Let $\alpha=\operatorname{diag}(a, \ldots, a, 1, \ldots, 1) \in G$, where the blocks of $a$ 's and 1's have respective lengths $n-2 k$ and $2 k$. Note that $\delta \in \alpha H$ and that conjugation by $\alpha$ fixes $\psi$. Thus

$$
{ }^{\delta}\left(\operatorname{Ind}_{G_{k}}^{H}(\psi)\right)={ }^{\alpha}\left(\operatorname{Ind}_{G_{k}}^{H}(\psi)\right)=\operatorname{Ind}_{G_{k}}^{H}\left({ }^{\alpha} \psi\right)=\operatorname{Ind}_{G_{k}}^{H}(\psi) .
$$

Therefore, $D^{n-2 k}$ stabilizes the equivalence class of $\operatorname{Ind}_{G_{k}}^{H}(\psi)$.

It follows from the preceding paragraphs that the group generated by $D^{n}$ and $D^{n-2 k}$ stabilizes the equivalence class of $\operatorname{Ind}_{G_{k}}^{H}(\psi)$. To complete the proof, note that this group is precisely $D^{d}$.

The Local Langlands Correspondence for $\operatorname{GL}(n)$ [4, 5] gives a bijection from the set of equivalence classes of irreducible representations of $G$ to a set consisting of certain $n$-dimensional complex representations of the Weil-Deligne group $W_{F}^{\prime}$ of $F$. The existence of the Langlands Correspondence for $\operatorname{SL}(n)$ follows from this by the work of Gelbart and Knapp 3]. Here the equivalence classes of irreducible 
representations of $H$ are parameterized by certain homomorphisms from $W_{F}^{\prime}$ to $\operatorname{PGL}(n, \mathbb{C})$. Moreover, in the case of $\operatorname{SL}(n)$, the correspondence is now many-toone; the fibers of the parameterization are the L-packets of $H$. In [3, Thm. 4.1], it is shown that the $L$-packets of $H$ coincide with the orbits of $G$ on equivalence classes of irreducible representations of $H$. Thus if $\rho$ is an irreducible admissible representation of $H$, the size of the $L$-packet containing $\rho$ is precisely $(G: G(\rho))=$ $(D: D(\rho))$. The following result gives a relationship between the Klyachko model of a representation $\rho$ of $H$ and the size of the $L$-packet containing $\rho$.

Corollary 2.1. Let $k$ be an integer, $0<2 k<n$. Let $d=(2 k, n)$. If the irreducible admissible representation $\rho$ of $H$ occurs in $\operatorname{Ind}_{G_{k}}^{H}\left({ }^{\gamma} \psi_{k}\right)$ for some $\gamma \in D$, then $D^{d} \subset$ $D(\rho)$. In particular, the size of the L-packet of $\rho$ is at most the index of $\left(F^{\times}\right)^{d}$ in $F^{\times}$. Thus if $d=1$, then $\rho$ must be stable; that is, the L-packet containing $\rho$ is a singleton.

Proof. Recalling that $D \cong F^{\times}$, the second and third statements follow immediately from the first, which we now verify. It follows from Lemma 2.3 that $D^{d}$ stabilizes $\operatorname{Ind}_{G_{k}}^{H}\left({ }^{\gamma} \psi_{k}\right)$. Let $\delta \in D^{d}$. Then $\rho$ occurs in $\operatorname{Ind}_{G_{k}}^{H}\left({ }^{\gamma \delta} \psi_{k}\right)$. By the uniqueness statement in Theorem 2.1, we must then have that $\gamma \delta \in \gamma D(\rho)$, so $\delta \in D(\rho)$.

\section{Self-Dual Representations}

Let $G$ be a totally disconnected locally compact group with $(\pi, V)$ an irreducible admissible representation of $G$, and $\iota$ a continuous automorphism of $G$ such that $\iota^{2}$ is the identity. Let $(\hat{\pi}, \hat{V})$ denote the smooth contragredient of $(\pi, V)$, where $\hat{V}$ is the smooth dual of $V$, and define the representation $\left({ }^{i} \pi, V\right)$ by ${ }^{\iota} \pi=\pi \circ \iota$. From Schur's Lemma, the representation $\pi$ satisfies ${ }^{\iota} \pi \cong \hat{\pi}$ if and only if there exists a nondegenerate bilinear form, unique up to scalar multiple, say $B: V \times V \rightarrow \mathbb{C}$, such that

$$
B\left(\pi(g) v,{ }^{\iota} \pi(g) w\right)=B(v, w) \text { for all } v, w \in V, g \in G .
$$

It follows that $B$ must be either symmetric, in which case we write $\varepsilon_{\iota}(\pi)=1$, or skew-symmetric, in which case we write $\varepsilon_{\iota}(\pi)=-1$. If ${ }^{\iota} \pi \neq \hat{\pi}$, then we let $\varepsilon_{\iota}(\pi)=0$. When $\iota$ is the trivial automorphism, then ${ }^{\iota} \pi=\pi \cong \hat{\pi}$ just means that $\pi$ is self-dual. In this case, we simply write $\varepsilon(\pi)$ for $\varepsilon_{\iota}(\pi)$. If $\pi$ is self-dual and $\varepsilon(\pi)=1$, we say that $\pi$ is orthogonal, and if $\varepsilon(\pi)=-1$, we say that $\pi$ is symplectic.

We begin with the following, which is a slight generalization of [13, Lemma 2.1]. Since the proof is virtually identical to the proof in [13, we just give an outline.

Lemma 3.1. Let $(\pi, V)$ be an irreducible, admissible, and unitarizable representation of the totally disconnected locally compact group $G$, and let $\iota$ be a continuous automorphism of $G$ such that $\iota^{2}$ is the identity. Then $\varepsilon_{\iota}(\pi)=1$ if and only if there exists a conjugate linear automorphism $\varphi: V \rightarrow V$ such that $\varphi^{2}=1$, and $\varphi\left({ }^{l} \pi(g) v\right)=\pi(g) \varphi(v)$ for all $v \in V$ and all $g \in G$.

Proof. Since $(\pi, V)$ is unitarizable, there is a positive definite Hermitian form $\langle\cdot, \cdot\rangle$ on $V$ which is $G$-invariant. First assume that there exists a conjugate linear automorphism $\varphi$ on $V$ with the above properties. If we define a bilinear form $B$ by $B(v, w)=\langle v, \varphi(w)\rangle$, then it follows that $B$ is nondegenerate and satisfies (3.1). To prove that $B$ is symmetric, it is enough to show that $\langle v, w\rangle=\overline{\langle\varphi(v), \varphi(w)\rangle}$, which follows from the uniqueness of $\langle\cdot, \cdot\rangle$ up to a positive scalar multiple. 
Conversely, suppose that $B$ is a nondegenerate symmetric form on $V$ which satisfies (3.1). Any element of the smooth dual $\hat{V}$ of $V$ is of the form $\langle\cdot, w\rangle$, for a unique $w \in V$. For any $w \in V$, the map $u \mapsto B(u, w)$ is a smooth linear functional of $V$, and so there is a unique $w^{\prime}$ such that $B(u, w)=\left\langle u, w^{\prime}\right\rangle$. This defines a conjugate linear map $w \mapsto w^{\prime}$ on $V$. Now, we must have $\langle v, w\rangle=\lambda \overline{\left\langle v^{\prime}, w^{\prime}\right\rangle}$, for all $v, w \in V$ and for some positive real number $\lambda$, by uniqueness of the Hermitian form $\langle\cdot, \cdot\rangle$. If we define $\varphi(v)=\sqrt{\lambda} v^{\prime}$, then $\varphi: V \rightarrow V$ has the desired properties.

The next result is a generalization of [13, Cor. 2.2], and we again use an argument very similar to the one appearing there.

Lemma 3.2. Let $(\pi, V)$ be an irreducible, admissible, and unitarizable representation of $G$, let $\iota$ be a continuous automorphism of $G$ such that $\iota^{2}$ is the identity, and let $H$ be a closed subgroup of $G$ which is stable under $\iota$. Let $\psi$ be a one-dimensional representation of $H$ such that ${ }^{\iota} \psi=\bar{\psi}$, and such that $\operatorname{dim}_{\mathbb{C}} \operatorname{Hom}_{H}(\pi, \psi)=1$. If ${ }^{\iota} \pi \cong \hat{\pi}$, then $\varepsilon_{\iota}(\pi)=1$.

Proof. Let $\langle\cdot, \cdot\rangle$ denote the $G$-invariant Hermitian form on $V$. We know that ${ }^{\iota} \pi \cong \hat{\pi}$, and we say that $T: V \rightarrow \hat{V}$ is the corresponding intertwining operator. There is also a conjugate linear isomorphism $L: V \rightarrow \hat{V}$ given by $L(w)=\langle\cdot, w\rangle$; note that $L$ satisfies $L(\pi(g) v)=\hat{\pi}(g) L(v)$ for all $g \in G, v \in V$. Then $\eta=L^{-1} \circ T$ is a conjugate linear automorphism of $V$ satisfying $\eta\left({ }^{\iota} \pi(g) v\right)=\pi(g) \eta(v)$ for all $g \in G, v \in V$. By Schur's lemma, we must have $\eta^{2}=\alpha$, where $\alpha$ is some nonzero complex scalar.

Now, let $\ell \in \operatorname{Hom}_{H}(\pi, \psi)$, with $\ell \neq 0$, and define $\tilde{\ell}: V \rightarrow \mathbb{C}$ by $\tilde{\ell}(v)=\overline{\ell(\eta(v))}$. Then, for any $h \in H, v \in V$, we have

$$
\tilde{\ell}(\pi(h) v)=\overline{\ell\left({ }^{\iota} \pi(h) \eta(v)\right)}=\overline{{ }^{\iota} \psi(h) \ell(\eta(v))}=\psi(h) \tilde{\ell}(v),
$$

since $\eta(\pi(h) v)={ }^{\iota} \pi(h) \eta(v)$ and ${ }^{\iota} \psi=\bar{\psi}$. So, $\tilde{\ell} \in \operatorname{Hom}_{H}(\pi, \psi)$, and we must have $\tilde{\ell}=\lambda \ell$ for some nonzero complex scalar $\lambda$. Since we then have $\ell(\eta(v))=\overline{\lambda \ell(v)}$ for all $v$, by substituting $\eta(v)$ for $v$ and from the fact that $\eta^{2}(v)=\alpha v$, we obtain $\alpha \ell(v)=\bar{\lambda} \lambda \ell(v)$. We now have $\alpha=\bar{\lambda} \lambda$, and we define $\varphi=\lambda^{-1} \eta$. Now, $\varphi: V \rightarrow V$ is a conjugate linear automorphism such that $\varphi^{2}=1$ and $\varphi\left({ }^{\iota} \pi(g) v\right)=\pi(g) \varphi(v)$ for all $g \in G, v \in V$. By Lemma 3.1, we have $\varepsilon_{\iota}(\pi)=1$.

If $(\pi, V)$ is an irreducible admissible representation of $G$, and $z$ is an element of the center of $G$, then it follows from Schur's lemma that $\pi(z)$ acts as a scalar on $V$, which we denote by $\omega_{\pi}(z)$. The next result follows directly from [15, Prop. 2].

Lemma 3.3. Let $s \in G$ such that $s^{2}=z$ is in the center of $G$. Define the automorphism $\iota$ on $G$ by $\iota(g)=s^{-1} g s$, so $\iota^{2}$ is the identity. Then for any irreducible admissible representation $(\pi, V)$ of $G$, we have $\varepsilon(\pi)=\omega_{\pi}(z) \varepsilon_{\iota}(\pi)$.

In [12, Sec. 3, Ex. (2)], Prasad describes when a generic self-dual representation of $\mathrm{SL}(n, F)$ is orthogonal and when it is symplectic (excluding the case that $n$ is 2 $\bmod 4$ and $F$ does not contain a square root of -1$)$. Here, we extend these results to include any self-dual irreducible admissible representation which is unitarizable.

Corollary 3.1. Let $F$ be a non-Archimedean local field of characteristic 0, and let $(\pi, V)$ be a self-dual, irreducible, admissible, and unitarizable representation of $H=\mathrm{SL}(n, F)$. Then

(1) If $n$ is odd or $n \equiv 0(\bmod 4)$, then $\varepsilon(\pi)=1$. 
(2) If $n \equiv 2(\bmod 4)$ and $F$ contains a square root of -1 , then $\varepsilon(\pi)=1$ if and only if the central element $-I$ of $\mathrm{SL}(n, F)$ acts trivially on $V$, that is, $\varepsilon(\pi)=\omega_{\pi}(-I)$.

Proof. By Theorem 2.1(2), there exists a $k, 0 \leq 2 k \leq n$, and a $\gamma \in D$, such that

$$
\operatorname{dim}_{\mathbb{C}} \operatorname{Hom}_{G}\left(\pi, \operatorname{Ind}_{\gamma_{G_{k}}}^{H}\left({ }^{\gamma} \psi_{k}\right)\right)=\operatorname{dim}_{\mathbb{C}} \operatorname{Hom}_{G_{k}}\left(\pi,{ }^{\gamma} \psi_{k}\right)=1 .
$$

First suppose that $2 k<n$, so that we may assume ${ }^{\gamma} G_{k}=G_{k}$.

If $n \equiv 0(\bmod 4)$, then define $s=\operatorname{diag}(-1,1, \ldots,-1,1)$; if $n \equiv 3(\bmod 4)$, then define $s=\operatorname{diag}(-1,1, \ldots, 1,-1)$; and if $n \equiv 1(\bmod 4)$, then define $s=$ $\operatorname{diag}(1,-1, \ldots,-1,1)$. In each case, $s \in H$ and $s^{2}=I$. If we define $\iota$ on $H$ by $\iota(g)=$ $s^{-1} g s$, then we must check that $G_{k}$ is stable under $\iota$. In general, if we conjugate the symplectic group defined by the skew-symmetric matrix $J$ by a matrix $a$, the result is the symplectic group defined by the skew-symmetric matrix $\left({ }^{t} a^{-1}\right) J\left(a^{-1}\right)$. In these cases, we are conjugating $\operatorname{Sp}(2 k, F)$, defined by $J_{k}$, by the diagonal matrix $s_{2 k}$ with entries given by the last $2 k$ entries of $s$. Since $s_{2 k} J_{k} s_{2 k}=J_{k}$ in each case, it is indeed true that $G_{k}$ is fixed by $\iota$. Now, we have ${ }^{\gamma} \psi_{k}\left(s^{-1} h s\right)=\bar{\gamma} \psi_{k}(h)$ for every $h \in G_{k}$, and ${ }^{\iota} \pi \cong \pi \cong \hat{\pi}$, since $\pi$ is self-dual. By Lemma 3.2. we have $\varepsilon_{\iota}(\pi)=1$, and by Lemma 3.3 we have $\varepsilon(\pi)=1$, as desired.

Now suppose that $n \equiv 2(\bmod 4)$, and that $F$ contains a square root of -1 , and say $\beta \in F$ such that $\beta^{2}=-1$. Define $s=\operatorname{diag}(\beta,-\beta, \ldots, \beta,-\beta)$, and define $\iota$ on $H$ by $\iota(g)=s^{-1} g s$. Then $s \in H$ and $s^{2}=-I$. In this case, defining $s_{2 k}$ as above, we have $s_{2 k}^{-1} J_{k} s_{2 k}^{-1}=-J_{k}$, and since the symplectic group defined by the skewsymmetric matrix $J_{k}$ is the same as the one defined by $-J_{k}$, we again have that $G_{k}$ is stable under $\iota$. As before, we have ${ }^{\gamma} \psi_{k}\left(s^{-1} h s\right)=\bar{\gamma} \psi_{k}(h)$ for every $h \in G_{k}$, and also ${ }^{l} \pi \cong \hat{\pi}$. By Lemmas 3.2 and 3.3 , we conclude that $\varepsilon(\pi)=\omega_{\pi}(-I)$.

In the case that $2 k=n$, we have that $\psi_{k}$ is trivial, so that ${ }^{s}\left({ }^{\gamma} \psi_{k}\right)=\psi_{k}$, but we must check that ${ }^{\gamma} G_{k}$ is fixed by $\iota$. In this case, if $\gamma=\operatorname{diag}(x, 1, \ldots, 1)$, then ${ }^{\gamma} G_{k}$ is the symplectic group defined by the skew-symmetric matrix $\tilde{J}=\left({ }_{A}{ }^{-A}\right)$, where $A$ is the $k$-by- $k$ matrix $\operatorname{diag}(x, 1, \ldots, 1)$. In each case for $\iota$ and $s$ above, for $n$ even, ${ }^{\iota}\left({ }^{\gamma} G_{k}\right)$ is the symplectic group defined by the skew-symmetric matrix $s^{-1} \tilde{J} s^{-1}$. It follows that ${ }^{\gamma} G_{k}$ is fixed by each $\iota$ by observing that this skew-symmetric matrix defines the same symplectic group as $\tilde{J}$.

Remarks. In [15, Sec. 6], the second-named author studies the values of $\varepsilon_{\iota}(\pi)$, where $\pi$ is an irreducible admissible representation of $\operatorname{GL}(n, F)$, and $\iota$ is the transpose-inverse automorphism composed with conjugation by the longest Weyl element. The statement in [15, Thm. 8] that $\varepsilon_{\iota}(\pi)=1$ for all such $\pi$ does not have a complete proof there. What is actually proved is that if $\pi$ is an irreducible admissible representation of $\operatorname{GL}(n, F)$, and there exists a character $\psi$ of the maximal unipotent subgroup such that ${ }^{\iota} \psi=\bar{\psi}$ and $\pi$ has a unique $\psi$-degenerate Whittaker model, then $\varepsilon_{\iota}(\pi)=1$. Also, the conclusion cannot be made in [15, Sec. 3] using similar methods that $\varepsilon_{\iota}(\pi)=1$ for every irreducible representation $\pi$ of the finite group $\operatorname{GL}\left(n, \mathbb{F}_{q}\right)$. However, this statement is already known to be true for the finite group $\operatorname{GL}\left(n, \mathbb{F}_{q}\right)$, while this is still an open question for the $p$-adic group $\operatorname{GL}(n, F)$.

For the statement in [15, Thm. 8] that $\varepsilon(\pi)=1$ for every self-dual, irreducible, admissible representation $\pi$ of $\mathrm{GL}(n, F)$, the proof is complete. It is possible that similar methods could be used to extend Corollary 3.1 to all self-dual irreducible admissible representations of $\mathrm{SL}(n, F)$. 


\section{ACKNOWLEDGMENTS}

Both authors thank the referee for suggestions and corrections to improve this paper and Alan Roche for helpful comments. The first-named author was supported by NSF grant DMS-0854844, and the second-named author was supported by NSF grant DMS-0854849.

\section{REFERENCES}

[1] D. Bump, Automorphic forms and representations, Cambridge Studies in Advanced Mathematics, 55, Cambridge University Press, Cambridge, 1997. MR.1431508 (97k:11080)

[2] C.J. Bushnell and P.C. Kutzko, The admissible dual of SL(N). I, Ann. Sci. École Norm. Sup. (4) 26 (1993), 261-279. MR.1209709 (94a:22033)

[3] S.S. Gelbart and A.W. Knapp, $L$-indistinguishability and $R$ groups for the special linear group, Adv. Math. 43 (1982), no. 2, 101-121. MR644669 (83j:22009)

[4] M. Harris and R. Taylor, The geometry and cohomology of some simple Shimura varieties, with an appendix by Vladimir G. Berkovich, Ann. of Math. Studies, 151, Princeton University Press, Princeton, New Jersey, 2001. MR.1876802 (2002m:11050)

[5] G. Henniart, Une preuve simple des conjectures de Langlands pour GL $(n)$ sur un corps p-adique, Invent. Math. 139 (2000), no. 2, 439-455. MR1738446(2001e:11052)

[6] M.J. Heumos and S. Rallis, Symplectic-Whittaker models for $G l_{n}$, Pacific J. Math. 146 (1990), no. 2, 247-297. MR1078382 (91k:22036)

[7] N.F.J. Inglis and J. Saxl, An explicit model for the complex representations of the finite general linear groups, Arch. Math. (Basel) 57 (1991), no. 5, 424-431. MR1129515|(92j:20039)

[8] A.A. Klyachko, Models for complex representations of the groups $\operatorname{GL}(n, q)$, Mat. Sb. (N.S.), 120(162) (1983), no. 3, 371-386. MR691984 (84j:20014)

[9] O. Offen and E. Sayag, On unitary representations of GL $(2 n)$ distinguished by the symplectic group, J. Number Theory 125 (2007), no. 2, 344-355. MR2332593 (2009a:22012)

[10] O. Offen and E. Sayag, Global mixed periods and local Klyachko models for the general linear group, Int. Math. Res. Not. IMRN (2008), Art. ID rnm 136, 25 pages. MR 2417789 (2009e:22017)

[11] O. Offen and E. Sayag, Uniqueness and disjointness of Klyachko models, J. Funct. Anal. 254 (2008), no. 11, 2846-2865. MR2414223(2009e:22018)

[12] D. Prasad, On the self-dual representations of a $p$-adic group, Int. Math. Res. Not. IMRN (1999), no. 8, 443-452. MR 1687319 (2000d:22019)

[13] D. Prasad and D. Ramakrishnan, Self-dual representations of division algebras and Weil groups: a contrast, preprint, 2008, available at www.math.tifr.res.in/ dprasad and www.math.caltech.edu/people/dinakar.html.

[14] M. Tadic̀, Notes on representations of non-Archimedean SL(n), Pacific J. Math. 152 (1992), no. 2, 375-396. MR.1141803 (92k:22029)

[15] C.R. Vinroot, Involutions acting on representations, J. Algebra 297 (2006), no. 1, 50-61. MR2206367 (2006j:20071)

Department of Mathematics and Statistics, American University, 4400 Massachusetts Avenue, NW, Washington, DC 20016

E-mail address: lansky@american.edu

Department of Mathematics, College of William and Mary, P. O. Box 8795, WilliamsBURG, VIRGINIA 23187

E-mail address: vinroot@math.wm.edu 